\title{
ORIGINAL ARTICLE Identification and treatment of sleep-disordered breathing in chronic spinal cord injury
}

\author{
A Sankari ${ }^{1,2}, \mathrm{JL}_{\text {Martin }}^{3,4}, \mathrm{AT}$ Bascom $^{2}, \mathrm{MN}$ Mitchell $^{3}$ and MS Badr ${ }^{1,2}$
}

Study design: A follow up on an ongoing prospective cohort study.

Objective: Spinal cord injury or disorder (SCI/D) patients have higher rates of sleep-disordered breathing (SDB) than the general population. The objectives of this study were to examine predictors of SDB diagnosis and to estimate rates of SDB treatment in SCI/D patients.

Setting: A SCl clinical sleep research laboratory.

Methods: Twenty-eight SCI/D patients (7 women, age 42.8 15.8 years; 16 cervical and 12 thoracic level injuries) completed a battery of questionnaires (Epworth Sleepiness Scale (ESS), Pittsburgh Sleep Quality Index (PSQI), Berlin questionnaire (BQ) and fatigue severity scale (FSS)) and had one night of attended laboratory polysomnography (PSG). Participants were then notified of the results of their PSG and were interviewed approximately 1 year later to assess clinical outcomes.

Results: The majority of patients reported poor sleep quality on all questionnaires. On the basis of PSG, 22 (79\%) patients had SDB (apnea-hypopnea index $(\mathrm{AHI}) \geqslant 5$ events per hour), and 17 (61\%) had moderate/severe SDB (AHI $\geqslant 15$ events per hour). Higher ESS scores were associated with a higher risk of $\mathrm{AHI} \geqslant 5$; however, other questionnaires did not distinguish between those with and without SDB using either AHI cutoff. In follow-up interviews, only 50\% of patients had spoken to a health-care provider about SDB and only six patients with SDB were prescribed treatment, four of whom were using the treatment at follow-up.

Conclusion: SDB is common and severe among SCI/D patients. Screening questionnaires do not appear to differentiate between those with and without SDB. Even when SDB was recognized, many patients remained untreated. The increased prevalence of cardiovascular disease in SCI/D patients could represent a consequence of untreated SDB, and improving diagnosis and management of SDB has the potential to improve outcomes for these patients.

Spinal Cord (2015) 53, 145-149; doi:10.1038/sc.2014.216; published online 16 December 2014

\section{INTRODUCTION}

Estimates suggest that $1.9 \%$ of the U.S. population or six million people report paralysis and 1275000 people have a spinal cord injury or disorder (SCI/D). ${ }^{1}$ In fact, SCI/D is the second most common cause for paralysis after stroke. ${ }^{2}$ Traumatic injuries (for example, motor vehicle accidents) are responsible for the majority of SCI/D. Today, people with SCI/D are living longer, and approximately $80 \%$ are 50 years of age and older. ${ }^{3}$ Increased life expectancy of the SCI/D population has been accompanied by an increased prevalence of chronic diseases common in older age including obesity, heart disease and metabolic disease. ${ }^{4}$ In fact, about two-thirds of patients with SCI/ $\mathrm{D}$ are overweight or obese and are, therefore, at increased risk for metabolic disorders, cardiac diseases and sleep-disordered breathing (SDB), as a result. ${ }^{5}$

Studies show that sleep disturbances are prevalent and sleep quality is significantly impaired in SCI/D patients. ${ }^{5-9}$ The underlying causes of poor sleep in SCI/D patients include depression, pain, bladder dysfunction, circadian misalignment, use of analgesics/hypnotics and SDB. ${ }^{9}$ The net effect is that individuals living with SCI/D suffer from sleep fragmentation, high snoring frequency and overall poorer sleep quality relative to able-bodied individuals. Sleep deficiency is related to many chronic health problems, including obesity, diabetes, hypertension and cardiovascular disease, all of which are common in SCI/D patients. ${ }^{10,11}$ Moreover, poor sleep may exacerbate other symptoms such as depressed mood and impaired cognitive performance, which are potential vulnerabilities in patients with SCI/D. ${ }^{12}$ Accordingly, sleep disturbance may be both a cause and a consequence of comorbid symptoms in SCI/D patients. Further research is needed to characterize better the sleep difficulties faced by these patients.

SDB is a common cause of sleep disruption in individuals living with SCI/D. The prevalence of SDB is two to fourfold higher than the general population. ${ }^{5,13-17}$ Recent reports have also found that more than half of SCI patients developed SDB in their first year post injury. ${ }^{18}$ Therefore, SCI/D may be an independent risk factor for the development of SDB. ${ }^{15}$ Nevertheless, the majority of SCI/D patients with SDB remain undiagnosed and untreated despite the established negative consequences of SDB and the high frequency of sleep-related complaints in SCI/D patients. ${ }^{5,19}$ One reason that SDB is critically important in these patients is that cardiopulmonary complications are major causes of morbidity and mortality in SCI/D patients because of decreased lung volume, ineffective cough, mucus retention and atelectasis. ${ }^{14,20}$ In fact, cardiovascular conditions have superseded

${ }^{1}$ Dingell VA Medical Center, Detroit, MI, USA; ${ }^{2}$ Department of Medicine, Wayne State University, Detroit, MI, USA; ${ }^{3}$ VA Greater Los Angeles Healthcare System, North Hills, CA, USA and ${ }^{4}$ David Geffen School of Medicine at the University of California, Los Angeles, CA, USA

Correspondence: Dr A Sankari, Division of Pulmonary, Critical Care and Sleep Medicine, 3990 John R, 3-Hudson, Detroit, MI 48201, USA

E-mail:atarbich@med.wayne.edu

Portions of these findings were presented at the American Professional Sleep Society meeting in Minnesota on June 2014 and reported in the form of abstract ${ }^{6}$

Received 26 March 2014; revised 27 October 2014; accepted 2 November 2014; published online 16 December 2014 
respiratory causes of death in cervical SCI/D patients and have become the most common cause for mortality in this disabled population. ${ }^{21}$ On the basis of the extensive literature linking untreated SDB to cardiovascular mortality, SDB may represent a treatable risk factor among SCI/D patients. ${ }^{22-24}$

The objectives of the current study were (i) to determine predictors of SDB among SCI/D patients based on commonly used sleep questionnaires and (ii) to gather information about clinical followup and positive airway pressure (PAP) therapy among SCI/D patients with SDB, once patients were informed of their diagnosis.

\section{MATERIALS AND METHODS}

\section{Subjects}

The Human Investigation Committee of Wayne State University and the John D. Dingell VA Medical Center (JDDVAMC) approved the study. Written informed consent was obtained from all participants, who completed overnight laboratory screening polysomnography (PSG). We studied adults ( $>18$ years old) with chronic SCI/D if they met the study inclusion and exclusion criteria. Inclusion criteria were (i) non-ventilator-dependent individuals with chronic SCI/D ( $>6$ months post injury) and (ii) American Spinal Injury Association grade $\mathrm{A}, \mathrm{B}, \mathrm{C}$ or $\mathrm{D},{ }^{25}$ with injuries at the cervical $(\mathrm{C} 4-\mathrm{C} 7)$ or thoracic level (T1T6). Exclusion criteria were (i) pregnant or lactating females; (ii) advanced heart failure, peripheral vascular disease or stroke; (iii) history of head trauma associated with loss of consciousness; (iv) advanced lung, liver or chronic kidney disease; and (v) extreme obesity, defined as body mass index $>38 \mathrm{~kg} \mathrm{~m}^{-2}$. Participants were recruited from local and regional SCI care centers including the JDDVAMC and the Rehabilitation Institute of Michigan.

\section{Measurements}

Every subject who agreed to enroll had a brief history and physical examination and then completed the following questionnaires: Epworth Sleepiness Scale (ESS), Pittsburgh Sleep Quality Index (PSQI), Berlin questionnaire (BQ) and Fatigue Severity Scale (FSS). The ESS and PSQI are validated self-administered questionnaires to assess daytime sleepiness and sleep quality, respectively. ${ }^{26-28}$ The ESS has eight items, and answers are scored 0-3 based on the self-rated likelihood of falling asleep in certain circumstances during the past month. A total score $\geqslant 10$ is considered clinically abnormal sleepiness. The PSQI is an 18-item index that measures seven areas of sleep quality: subjective sleep quality, sleep latency, sleep duration, habitual sleep efficiency, sleep disturbances, use of sleep medications and daytime dysfunction over the last month. ${ }^{26,27}$ Each of these seven domains is composed of a $0-3$ scale, whereby 3 reflects the negative extreme on the scale. A total score $>5$ indicates poor sleep quality. The FSS is a self-administered questionnaire to measure the level of agreement with fatigue symptom statements for the prior week. ${ }^{29}$ Scoring of level of agreement is based on a $0-7$ scale, whereby 7 reflects strong agreement, and scores $\geqslant 2$ indicate significant fatigue. The $\mathrm{BQ}$ is a self-administered questionnaire consisting of three categories related to the risk of having sleep apnea. Patients can be classified into 'High Risk' or 'Low Risk' based on their responses. Individuals who endorse two out of the three categories are considered 'high risk' for SDB. ${ }^{30}$

Standard laboratory PSG was performed according to the standards of the American Academy of Sleep Medicine, and respiratory events were scored using the 2012 AASM recommended scoring criteria. ${ }^{31}$ Hypopnea events were scored with EEG arousals even if the $4 \%$ desaturation threshold was not reached. SDB was defined as an apnea-hypopnea index (AHI) of $\geqslant 5$ events per hour of sleep. In addition to standard full attended PSG (including EEG, EOG and chin EMG), nasal airflow was measured by a pneumotachometer (Hans Rudolph, Inc., Model 3700A, Shawnee, KS, USA) connected to a tight-fitting nasal mask to enhance the quality of the respiratory signals during the recording.

After completing the PSG, all participants with $\mathrm{AHI} \geqslant 5$ events per hour were notified verbally (personal communication or telephone call from study physician or research coordinator) and were mailed a letter describing their abnormal findings. This is standard practice in our research studies of SDB. Participants with SDB were provided with specific instructions to follow up with their own health-care provider for clinical evaluation and treatment of
Table 1 Follow-up survey for patients with sleep-disordered breathing

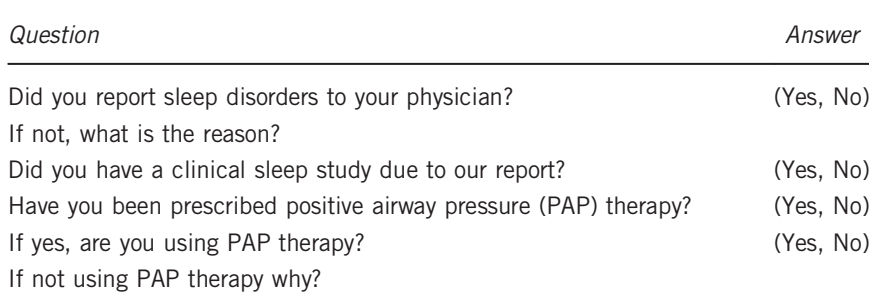

SDB. When possible (and with the patient's permission), the patient's primary care provider was notified. For the purposes of this study, a follow-up phone call was made to all participants with AHI $\geqslant 5$ at least 6 months (on average, 12 months) after notifying them of the results of their PSG. The goal of these calls was to assess the outcomes of informing the patient about the presence of SDB using the procedures described above. Of the 22 individuals with SDB, two participants' phones had been disconnected and they could not be reached. One participant stated he did not receive a letter, so it was re-sent. During this follow-up call, a short interview was performed (see Table 1 for a list of interview questions).

\section{Data analysis}

Descriptive statistics, in the form of frequencies (for binary and nominal variables) and means and standard deviations (for continuous variables), were computed for the overall sample, and then separately for those with SDB $(\mathrm{AHI} \geqslant 5)$ vs those without $\mathrm{SDB}(\mathrm{AHI}<5)$. We also compared individuals with $\mathrm{AHI} \geqslant 15$ to those with $\mathrm{AHI}<15$. $T$-tests were used for continuous variables and Fisher's exact tests for binary/nominal variables.

Bivariate analyses were performed using two outcome variables. First, we compared those with and without SDB using a cutoff of $\mathrm{AHI} \geqslant 5$ ) and second we used a cutoff of $\mathrm{AHI} \geqslant 15$. The seven core candidate predictors used to predict each outcome were (1) scoring as 'high risk' on the BQ, (2) having a high ESS score ( $\geqslant 10)$, (3) ESS total score, (4) having a high PSQI score $(>5)$, (5) PSQI total score, (6) having a high FSS ( $\geqslant 2$ ) and (7) FSS total score. Three additional predictors were considered as well, (8) age, (9) body mass index and (10) neck circumference. For the binary predictors (that is, 1, 2, 4 and 6), Fisher's exact tests were performed examining the association between SDB and the predictor. Exact logistic regression analyses were performed for the continuous predictors (that is, 3, 5, 7, 8, 9 and 10). All analyses were performed using Stata version 13.1 (StataCorp. 2013, Stata Statistical Software, College Station, TX, USA) and the exact logistic regression analyses were performed using the 'exlogistic' command. ${ }^{32}$

\section{RESULTS \\ Participant characteristics}

We studied 28 chronic SCI/D participants (21 males; age $42.8 \pm 15.8$ years; body mass index $26.2 \pm 4.9$; neck circumference $39.4 \pm 4$; 16 cervical and 12 thoracic levels). The etiologies of SCI/D were mainly due to gun shots $(36 \%)$ or motor vehicle accidents (32\%; see Table 2 ).

\section{Patient-reported sleep disturbances}

Using four screening sleep health questionnaires (ESS, PSQI, FSS and $\mathrm{BQ}$ ) the majority of SCI/D patients reported poor sleep quality (see Table 3). Overall, $60.7 \%$ had ESS scores of 10 or more (mean $\mathrm{ESS}=10.5 \pm 4.3)$ indicating sleepiness, $85.7 \%$ had PSQI scores more than 5 (mean PSQI $=10.5 \pm 4.3$ ) indicating poor sleep quality, $96.4 \%$ had FSS scores of 2 or more (mean FSS $=4.5 \pm 1.5$ ) indicating significant fatigue, and $51.9 \%$ had high-risk for SDB, based on the BQ.

\section{Severity of SDB}

On the basis of the attended laboratory PSG, the mean AHI was 29.8 (s.d. $=24.7)$. Of the 28 patients studied, 79\% $(n=22)$ had SDB 
Table 2 Participant characteristics for the overall sample (overall) and separately for $\mathrm{AHI}<5$ (vs $\mathrm{AHI} \geqslant 5$ ) and $\mathrm{AHI}<15$ (vs $\mathrm{AHI} \geqslant 15$ )

\begin{tabular}{|c|c|c|c|c|c|c|c|c|}
\hline & Overall & $A H I<5$ & $A H I \geqslant 15$ & P-value & $A H I<15$ & $A H I \geqslant 15$ & P-value & N \\
\hline Injury location & & & & 0.057 & & & 0.019 & 28 \\
\hline Cervical (C4-C7) & $57.1 \%(16)$ & $16.7 \%(1)$ & $68.2 \%(15)$ & & $27.3 \%$ (3) & $76.5 \%(13)$ & & \\
\hline Age (years) & $42.8(15.8)$ & $31.0(7.6)$ & $46.0(16.0)$ & 0.037 & $36.5(14.6)$ & 46.9 (15.6) & 0.088 & 28 \\
\hline BMI $\left(\mathrm{kg} \mathrm{m}^{-2}\right)$ & $26.2(4.9)$ & $25.9(3.6)$ & $26.3(5.3)$ & 0.840 & $26.4(4.6)$ & $26.1(5.3)$ & 0.890 & 28 \\
\hline Gender (male) & $75.0 \%(21)$ & $50.0 \%(3)$ & $81.8 \%(18)$ & 0.144 & $45.5 \%(5)$ & $94.1 \%(16)$ & 0.007 & 28 \\
\hline Etiology & & & & 0.039 & & & 0.077 & 28 \\
\hline Gun shot & $35.7 \%(10)$ & $83.3 \%(5)$ & $22.7 \%(5)$ & & $63.6 \%(7)$ & $17.6 \%$ (3) & & \\
\hline Motor vehicle accident & $32.1 \%(9)$ & $0.0 \%(0)$ & $40.9 \%(9)$ & & $18.2 \%(2)$ & $41.2 \%(7)$ & & \\
\hline Diving/fall & $10.7 \%(3)$ & $0.0 \%(0)$ & $13.6 \%(3)$ & & $0.0 \%(0)$ & $17.6 \%(3)$ & & \\
\hline Surgical/other & $21.4 \%(6)$ & $16.7 \%(1)$ & $22.7 \%(5)$ & & $18.2 \%(2)$ & $23.5 \%(4)$ & & \\
\hline
\end{tabular}

Abbreviations: AHI, apnea-hypopnea index; BMI, body mass index; NC, neck circumference.

Table 3 Comparison of groups using clinical cutoff scores on sleep health questionnaires

\begin{tabular}{|c|c|c|c|c|c|c|c|c|}
\hline & Overall & $A H I<5$ & $A H I \geqslant 5$ & P-value & $A H I<15$ & $A H I \geqslant 15$ & P-value & N \\
\hline$E S S \geqslant 10$ & $60.7 \%(17)$ & $33.3 \%$ (2) & $68.2 \%(15)$ & 0.174 & $54.5 \%(6)$ & $64.7 \%(11)$ & 0.701 & 28 \\
\hline PSQI > 5 & $85.71 \%(24)$ & $100.0 \%(6)$ & $81.8 \%(18)$ & 0.549 & $81.8 \%(9)$ & $88.2 \%(15)$ & 1.0 & 28 \\
\hline$B Q=$ high risk & $51.9 \%(14)$ & $16.7 \%(1)$ & $61.9 \%(13)$ & 0.077 & $30.0 \%(3)$ & $64.7 \%(11)$ & 0.120 & 27 \\
\hline
\end{tabular}

Abbreviations: AHI, apnea-hypopnea index; BQ, Berlin questionnaire; ESS, Epworth Sleepiness Scale; FSS, Fatigue Severity Scale; PSQI, Pittsburgh Sleep Quality Index.

$(\mathrm{AHI} \geqslant 5)$ and $61 \%(n=17)$ had moderate to severe $\mathrm{SDB}(\mathrm{AHI} \geqslant 15)$. A majority of SCI patients $(77.3 \%$, that is, 17/22) with SDB had $\mathrm{AHI} \geqslant 15$ events per hour indicating a moderate to severe degree of SDB. It is notable that only four participants had previously been diagnosed with SDB before participating in the study.

\section{Differences between individuals with and without SDB}

Table 2 shows comparisons between patients with and without SDB (using an AHI cutoff of 5) and between patients with and without moderate/severe SDB (using an AHI cutoff of 15). There were significant differences between those with $\mathrm{AHI} \geqslant 5$ vs $<5$ in terms of the underlying SCI/D etiology. Second, as we have previously reported those with cervical SCI had a higher prevalence of moderate to severe SDB than those with thoracic injuries, and males were more likely to have moderate to severe SDB than females. ${ }^{5,15}$ There were no other significant differences between patients with and without SDB using either AHI cutoff score.

In the series of bivariate analyses predicting the presence vs absence of SDB, 2 of the 10 predictors distinguished between those with and without $\mathrm{SDB}(\mathrm{AHI} \geqslant 5$ vs $\mathrm{AHI}<5)$ : $\mathrm{ESS}$ total score $(\mathrm{OR}=1.28$, $P=0.048)$ and age $(\mathrm{OR}=1.12, P=0.025)$. For a one unit increase in ESS score, the odds of having an $\mathrm{AHI} \geqslant 5$ increased by $28 \%$. A 1 year increase in age was associated with a $12 \%$ increase in the odds of having an $\mathrm{AHI} \geqslant 5$. None of the other eight predictors was significantly associated with $\mathrm{SDB}(\mathrm{AHI} \geqslant 5$ vs $\mathrm{AHI}<5)$. Furthermore, none of the 10 predictors was significantly associated with moderate to severe SDB $(\mathrm{AHI} \geqslant 15$ vs $\mathrm{AHI}<15)$.

\section{Outcomes of SDB diagnosis}

Figure 1 depicts rates of PAP therapy acceptance and use among the 18 SCI/D patients who were notified about their SDB diagnosis using the procedures describe above and the four individuals who were previously diagnosed with SDB (total $n=22$ ). Out of the 20 individuals we were able to contact by phone, we found that only $10(50 \%)$ had discussed SDB with a health-care provider. Among those who did not $(n=10)$, the most common reason given was that the patient did not see sleep difficulties as a significant concern worthy of clinical attention $(n=6)$. Furthermore, a clinical PSG was not conducted for 2 out of 10 patients who did speak to a provider because of the patient's perception that he/she would not be able to tolerate PAP therapy. The remaining six individuals were prescribed PAP therapy, and a subset of four reported using it at the time of the follow-up interview. The two individuals not using PAP therapy indicated that it was too uncomfortable to tolerate.

\section{DISCUSSION}

This study expands our prior work in the area of sleep quality and SDB among patients with SCI/D. ${ }^{5,15}$ The main findings of this study are (i) the majority of SCI/D patients reported poor sleep quality on multiple sleep questionnaires; (ii) individuals with SCI/D typically had moderate to severe SDB; (iii) among the sleep questionnaires examined (ESS, PSQI, FSS and BQ), higher ESS scores were associated with elevated risk of SDB, but questionnaires generally did not distinguish between patients with and without SDB; (iv) Even when SDB was diagnosed in patients with SCI/D, clinical management of the disorder remains a challenge.

Sleep quality is poor for patients living with SCI/D

The majority of patients with SCI/D in our study reported poor sleep quality, regardless of the presence or absence of SDB. Despite the poor sleep quality measured by all four screening sleep health questionnaires (ESS, PSQI, FSS and BQ), only ESS predicted the presence of 


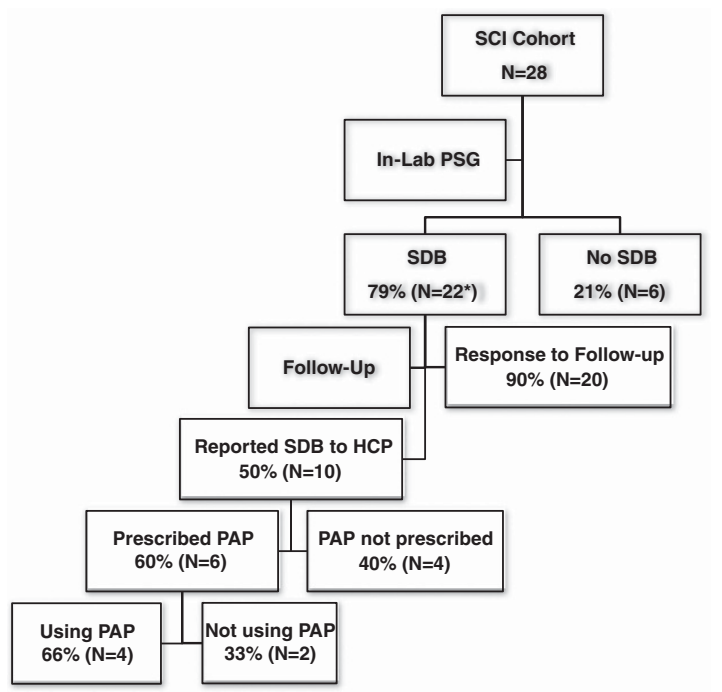

Figure $1 \mathrm{~A}$ flow chart to illustrate the rate of PAP therapy acceptance and tolerance in SCI/D patients with SDB diagnosis. HCP: healthcare provider; PAP: positive airway pressure; PSG: polysomnography; SDB: Sleepdisordered breathing.

SDB defined by $\mathrm{AHI} \geqslant 5$. The ESS questionnaire is a commonly used measure to determine the presence and severity of subjective excessive daytime sleepiness. It is possible that reported sleepiness is associated with SDB, but other types of sleep-related problems (for example, insomnia) can contribute to sleepiness in SCI/D patients as well. This finding is similar to previous studies in sleep disorders clinic patients using the ESS, which was useful in identified patients with and without SDB in clinical settings; ${ }^{28}$ however, unlike prior research, we did not find the cutoff score of 10 to be useful, as approximately one-third of individuals without SDB scored about this threshold, and about onethird of individuals with SDB scored below this threshold. In addition, none of the sleep questionnaires (including ESS) distinguished between those with and without moderate to severe SDB $(\mathrm{AHI} \geqslant 15$ vs $\mathrm{AHI}<15$ ). This could be because of the fact that the majority of SCI/D patients in our study had moderate or severe SDB, the reported sleep disturbances being so severe, or because of small sample size. The BQ, which is specially designed to identify individuals at high risk for SDB, also misclassified a significant proportion of patients. It appears the BQ is neither sensitive nor specific as a screening tool in SCI/D patients. Future research and larger studies are needed to determine whether sleep health questionnaires can be refined and adapted for use in the SCI/D population.

In contrast to other reports, the rate of SDB found in our study was higher than what has been observed in other studies. ${ }^{3,13,18}$ This may be due to several factors. First, we used in-lab attended PSG with pharyngeal catheter and pneumotachometer to accurately detect respiratory events. This may have enabled us to identify more events than were associated using other methodologies for recording respiratory variables during PSG. Second, we scored arousals and associated respiratory events independent of desaturation because patients with SCI/D may experience an arousal in the absence of a desaturation during a respiratory event during sleep. Third, our SCI/D population may have had more long-standing comorbidities than patients studied previously. We also found that moderate to severe SDB was more predominant in men with cervical SCI/D, which is not surprising as male gender and tetraplegia are both considered risk factors for SDB. ${ }^{15}$ These are important considerations, as home sleep testing may be an attractive alternative to laboratory studies in patients with SCI/D; however, the higher rates found in our study suggests that more sophisticated diagnostic testing may enhance sensitivity of testing and enable detection of more subtle forms of SDB.

Although we were not able to clinically follow these patients and make direct referrals to a sleep disorders specialist for clinical management, we did use our standard process for notifying patients (verbally and in writing), and when possible notifying their primary care providers. We also gathered follow-up information via interview from patients about why they did or did not choose to follow-up with their own providers about SDB. Our findings from these semistructured interviews suggest that, even when diagnosed, there are significant barriers to treating SDB among patients with SCI/D. First, patients felt that, given the host of medical conditions with which they were coping, SDB was not sufficiently impactful to warrant attention from their clinical providers. Second, when offered treatment, they experienced the same difficulties commonly reported by able-bodied individuals who are new users of PAP therapy including discomfort from the equipment. ${ }^{33}$ Taken together, these findings suggest that targeted patient education combined with support at PAP initiation may be useful for SCI/D patients.

In summary, the rates of SDB are high among patients with SCI/D, and may remain unrecognized and untreated despite the high prevalence of the disease and the known associated cardiovascular risks. It does not appear that routine screening with sleep questionnaires can identify individuals with moderate to severe disease, and development of new screening tools may be required. In addition, there are significant challenges to the treatment of patients who are identified as having SDB. Improved screening and detection of SDB should be combined with strategies to increase the acceptability of and adherence to PAP therapy in this particularly vulnerable patient group. ${ }^{34-37}$

\section{DATA ARCHIVING}

There were no data to deposit.

\section{CONFLICT OF INTEREST}

The authors declare no conflict of interest.

\section{ACKNOWLEDGEMENTS}

The research was supported by the United States Department of Veterans Affairs Merit Review \# 1I01CX001040 and Career Development Award \# 1IK2CX000547 from the Clinical Science Research \& Development Service of the VA Office of Research and Development.

\section{DISCLOSURE}

This was not an industry-supported study.

1 Reeve Foundation Paralysis Resource Center. One Degree of Separation: Paralysis and Spinal Cord Injury in the United States. http://www.christopherreeve.org/site/c mtKZKgMWKwG/b.5184189/k.5587/Paralysis_Facts_Figures.htm.

2 Jackson A, Dijkers M, DeVivo M, Poczatek R. A demographic profile of new traumatic spinal cord injuries: Change and stability over 30 years. Arch Phys Med Rehabil 2004; 11: $1740-1748$.

3 Short D, Stradling J, Williams S. Prevalence of sleep apnoea in participants over 40 years of age with spinal cord lesions. J Neurol Neurosurg Psychiatry 1992; 55 1032-1036.

4 Weaver F. M, Collins E G, Kurichi J, Miskevics S, Smith B, Rajan S, Gater D. Prevalence of obesity and high blood pressure in veterans with spinal cord injuries and disorders: a retrospective review. Am J Phys Med Rehabil 2007; 86: 22-29.

5 Sankari A, Bascom A, Oomman S, Badr MS. Sleep disordered breathing in chronic spinal cord injury. J Clin Sleep Med 2014; 10: 65-72. 
6 Sankari A, Bascom A, Martin J, Badr S. Health care disparities in the diagnosis and treatment of sleep disordered breathing in patients with spinal cord injury. SLEEP 2014; 37(Abstract Suppl): A237.

7 Widerstrom-Noga EG, Felipe-Cuervo E, Yezierski RP. Chronic pain after spinal injury: interference with sleep and daily activities. Arch Phys Med Rahabil 2001; 82: 1571-1577.

8 Budh CN, Hultling C, Lundeberg T. Quality of sleep in individuals with spinal cord injury: a comparison between patients with and without pain. Spinal Cord 2005; 43 85-95.

9 Biering-Sørensen F, Biering-Sørensen M. Sleep disturbances in the spinal cord injured: an epidemiological questionnaire investigation, including a normal population. Spinal Cord 2001; 39: 505-513.

10 Spiegel K, Knutson K, Leproult R, Tasali E, Cauter EV. Sleep loss: a novel risk factor for insulin resistance and Type 2 diabetes. J App/ Physiol 2005; 99: 2008-2019.

11 Ayas NT, White DP, Manson JE, Stampfer MJ, Speizer FE, Malhotra A, Hu FB. A prospective study of sleep duration and coronary heart disease in women. Arch Intern Med 2003; 163: 205-220.

12 Pilcher JJ, Huffcutt AJ. Effects of sleep deprivation on performance: A meta-analysis. Sleep 1996; 19: 318-326.

13 Leduc B, Dagher J, Mayer P, Bellemare F, Lepage Y. Estimated prevalence of obstructive sleep apnea-hypopnea syndrome after cervical cord injury. Arch Phys Med Rehabil 2007; 88: 333-337.

14 Levi R, Hultling C, Nash M, Seiger A. The Stockholm spinal cord injury study: Medical problems in a regional SCl population. Paraplegia 1995; 33: 308-315.

15 Sankari A, Bascom-Latin A, Chowdhuri S, Badr MS. Tetraplegia is a risk factor for central apnea. J Appl Physiol 2014; 116: 345-353.

16 Star A, Osterman A. Sleep apnea syndrome after spinal cord injury. Report of a case and literature review. Spine 1988; 13: 116-117.

17 Stockhammer E, Tobon A, Michel F. Characteristics of sleep apnea syndrome in tetraplegic participants. Spinal Cord 2002; 40: 286-294.

18 Berlowitz D, Brown D, Campbell D, Pierce R. A longitudinal evaluation of sleep and breathing in the first year after cervical spinal cord injury. Arch Phys Med Rehabil 2005; 86: 1193-1199.

19 Jennum P, Ibsen R, Kjellberg J. Morbidity prior to a diagnosis of sleep-disordered breathing: a controlled national study. J Clin Sleep Med 2013; 9: 103-108.

20 Teasell AP, Arnold AV, Krassioukov A, Delaney GA. Cardiovascular consequences of loss of supraspinal control of the sympathetic nervous system following spinal cord injuries. Arch Phys Med Rehabil 2000; 81: 506-516.

21 Myers J, Lee M, Kiratli J. Cardiovascular disease in spinal cord injury: an overview of prevalence, risk, evaluation, and management. Am J Phys Med Rehabil 2007; 86: $142-152$.

22 Somers VK, White DP, Amin R, Abraham WT, Costa F, Culebras A et al. Sleep apnea and cardiovascular disease. J Am Coll Cardiol 2008; 52: 686-717.
23 Marin JM, Carrizo SJ, Vicente E, Agusti AG. Long-term cardiovascular outcomes in men with obstructive sleep apnoea-hypopnoea with or without treatment with continuous positive airway pressure: an observational study. Lancet 2005; 365: 1046-1053.

24 Shahar E, Whitney CW, Redline S, Lee ET, Newman AB, Javier Nieto F et al. Sleep-disordered breathing and cardiovascular disease: cross-sectional results of the Sleep Heart Health Study. Am J Respir Crit Care Med 2001; 163. 19-25.

25 ASIA Impairment Scale, clinical syndromes, and standard neurological classification of spinal cord injury. http://www.asia-spinalinjury.org/publications/index.html.

26 Buysse DJ, Reynolds CF III, Monk TH, Berman SR, Kupfer DJ. The Pittsburgh Sleep Quality Index: A new instrument for psychiatric practice and research. Psychiatry Res 1989; 28: 193-213.

27 Cole JC, Motivala SJ, Buysse DJ, Oxman MN, Levin MJ, Irwin MR. Validation of a 3factor scoring model for the Pittsburgh Sleep Quality Index in older adults. Sleep 2006; 29: $112-116$.

28 Johns MW. Daytime sleepiness, snoring, and obstructive sleep apnea. The Epworth sleepiness scale. Chest 1993; 103: 30-36.

29 Krupp LB, LaRocca NG, Muir-Nash J, Steinberg AD. The fatigue severity scale. Application to patients with multiple sclerosis and systemic lupus erythematosus. Arch Neurol 1989; 46: 1121-1123.

30 Netzer NC, Stoohs RA, Netzer CM, Clark K, Strohl KP. Using the Berlin Questionnaire to identify patients at risk for a sleep apnea syndrome. Ann Intern Med 1999; 131: 485-491.

31 Berry R, Brooks R, Gamaldo C, Harding S, Marcus C, Vaughn B for the American Academy of Sleep Medicine. The AASM Manual for the Scoring of Sleep and Associated Events: Rules, Terminology and Technical Specifications, Version 2.0. American Academy of Sleep Medicine: Darien, IL, USA, 2012. www.aasmnet.org.

32 StataCorp. Stata Statistical Software: Release 13. StataCorp LP: College Station, TX, USA, 2013.

33 Weaver TE, Grunstein R. Adherence to continuous positive airway pressure therapy. Proc Am Thorac Soc 2008; 5: 173-178.

34 Sajkov D, Marshall R, Walker P, Mykytyn I, McEvoy RD, Wale J, Flavell H, Thornton A T, Antic R. Sleep apnoea related hypoxia is associated with cognitive disturbances in patients with tetraplegia. Spinal Cord 1998; 36: 231-239.

35 lezzoni LI, McCarthy EP, Davis RB, Siebens H. Mobility impairments and use of screening and preventive services. Am J Public Health 2000; 90: 955-961.

$36 \mathrm{McNicholas} \mathrm{WT}$, Bonsigore MR. Sleep apnoea as an independent risk factor for cardiovascular disease: current evidence, basic mechanisms and research priorities. Eur Respir J 2007; 29: 156-178.

37 Wallace D M, Ramos A R, Rundek T. Sleep disorders and stroke. Int J Stroke 2012; 7: 231-242. 